\title{
Two further generalizations of the Calkin-Wilf tree
}

\author{
Toufik Mansour and Mark Shattuck
}

In this paper, we consider two further analogues of the CalkinWilf tree and of the Calkin-Wilf sequence. We first consider $(p, q)$ versions of these whereby we show that a two-variable generalization of the latter is given, equivalently, in terms of a generalization of the former. In particular, we show that the sequence of $(p, q)$-generating functions counting the hyperbinary expansions of $n$ according to the total number of distinct powers used and the number of powers used twice arises as the sequence of numerators for the rational functions which label the vertices of our $(p, q)$ Calkin-Wilf tree. We also define a $k$-dimensional $q$-generalization of the Calkin-Wilf tree and of the Calkin-Wilf sequence. Having defined the $n$-th term of the latter in terms of the generating function counting the hyper $k$-expansions of $n$ according to the number of powers that are used exactly $k$ times, we show that it is given equivalently in terms of the former.

AMS 2000 SUBJECT CLASSIFICATIONS: Primary 05A30; secondary 05A15. KEYwORDS AND PHRASES: Calkin-Wilf tree, Calkin-Wilf sequence, hyperbinary expansion, $q$-analogue.

\section{Introduction}

The Calkin-Wilf tree is a binary tree in which each vertex is assigned a positive rational number. It is defined recursively as follows: The root of the tree is 1 , and each vertex $\frac{a}{b}$ has two children, namely $\frac{a}{a+b}$ (the left child) and $\frac{a+b}{b}$ (the right one). See Figure 1 below.

Calkin and Wilf [5] have shown that each positive rational number appears exactly once in this tree, as a fraction in lowest terms. Reading the tree line-by-line and left-to-right, the Calkin-Wilf sequence starts with

$$
\frac{1}{1}, \frac{1}{2}, \frac{2}{1}, \frac{1}{3}, \frac{3}{2}, \frac{2}{3}, \frac{3}{1}, \frac{1}{4}, \frac{4}{3}, \frac{3}{5}, \frac{5}{2}, \frac{2}{5}, \frac{5}{3}, \frac{3}{4}, \frac{4}{1}, \ldots
$$

Reznick [9] mentioned that this sequence was investigated by Stern [10] as early as 1858, and Newman (see Knuth [8]) found that this sequence satisfies 


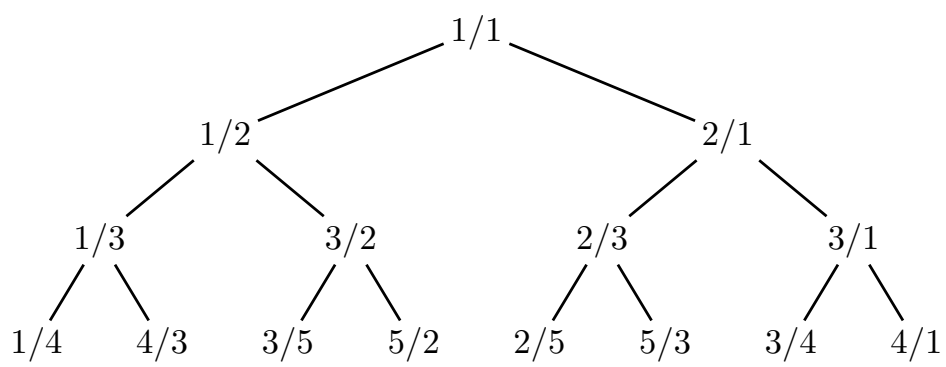

Figure 1: The first four levels of the Calkin-Wilf tree.

the somewhat unusual recurrence

$$
x_{n+1}=\frac{1}{2\left\lfloor x_{n}\right\rfloor+1-x_{n}}, \quad n \geq 1,
$$

with initial condition $x_{1}=1$.

The Calkin-Wilf sequence has many interesting properties. For example, the sequence of numerators gives the number of hyperbinary representations of all the nonnegative integers (see [5]). It can be used as a model for the game Euclid, first formulated by Cole and Davie [6] (see also Hofmann et al. [7]). Alkauskas and Steuding [2] considered several statistical properties of the Calkin-Wilf tree, and Bates and Mansour [4] have recently presented a $q$-version of it.

If $k \geq 2$, then a hyper $k$-expansion of the number $n$ is one consisting of powers of $k$ in which a given power can appear at most $k$ times. Hyperbinary will describe the case $k=2$. We consider two related sequences of polynomials. The first, denoted $f(n ; p, q)$, is the sequence of generating functions counting the hyperbinary expansions of $n$ according to the total number of distinct powers used and the number of powers used twice. The second, denoted $f_{k}(n ; q)$, is the sequence of generating functions counting the hyper $k$-expansions of $n$ according to the number of powers that are used exactly $k$ times, where $k \geq 2$ is fixed. Note that the former sequence of polynomials reduces to the number of hyperbinary expansions of $n$ when $p=q=1$, while the later reduces to it when $k=2$ and $q=1$.

We show that the sequence of fractions $\left\{\frac{f(n ; p, q)}{f(n+1 ; p, q)}\right\}$ provides a $(p, q)$ generalization of the Calkin-Wilf sequence, which may be expressed, equivalently, in terms of a $(p, q)$-Calkin-Wilf tree wherein each vertex is assigned instead a rational function in $p$ and $q$, see Theorem 2.2 below. We also present a $(p, q)$-generalization of (1) above which reduces to it when $p=q=1$. Furthermore, we consider a $k$-dimensional $q$-analogue of the Calkin-Wilf tree in 


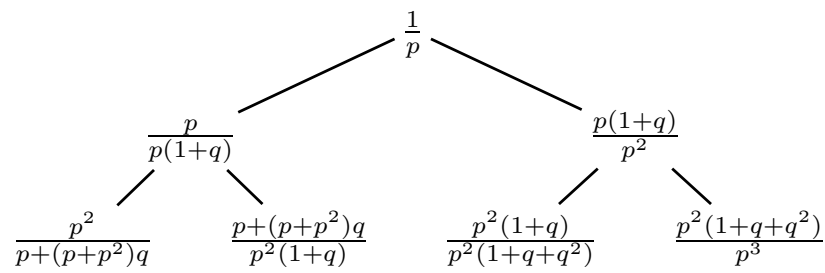

Figure 2: The first three levels of the $(p, q)$-Calkin-Wilf tree.

which each vertex is now labelled by a vector of fixed length $k$ whose entries are polynomials in the indeterminate $q$ and show in Theorem 3.2 below that this tree gives the sequence of polynomials $f_{k}(n ; q)$.

In what follows, we let $\mathbb{N}_{0}$ and $\mathbb{N}$ denote the nonnegative and positive integers, respectively. Let $\mathbb{R}$ denote the set of real numbers and $\mathbb{R}^{+}$its positive members. Empty sums take the value zero and empty products the value one, with $0^{0}=1$.

\section{The $(p, q)$-Calkin-Wilf tree and sequence}

The following definition of the $(p, q)$-Calkin-Wilf tree extends the definition of the Calkin-Wilf tree found in Bates et al. [3].

Definition 2.1 $((p, q)$-Calkin-Wilf tree). The $(p, q)$-Calkin-Wilf tree is a binary tree with root $\frac{1}{p}$. Each vertex $\frac{a}{b}$ from level 1 onwards is a parent to two children: a left child $\frac{p a}{p q a+b}$ and a right child $\frac{p q a+b}{p b}$, where $p$ and $q$ may either be regarded as indeterminates or as elements of $\mathbb{R}^{+}$. Each of these children is located one level below its parent on the tree.

Figure 2 shows the first three levels of the $(p, q)$-Calkin-Wilf tree.

Clearly, by induction on $j$, the leftmost and rightmost vertices in level $j$ of the $(p, q)$-Calkin-Wilf tree are, respectively,

$$
\frac{p^{j-1}}{p+\left(p+p^{2}+\cdots+p^{j-1}\right) q} \quad \text { and } \quad \frac{p^{j-1}\left(1+q+\cdots+q^{j-1}\right)}{p^{j}} .
$$

We next define $(p, q)$-generalizations for the concepts of hyperbinary expansion and sequence.

Definition 2.2 (( $p, q)$-hyperbinary expansions). The hyperbinary expansion of a number $n$ is an expansion of $n$ as a sum of powers of 2, each power being used at most twice. We denote the set of all hyperbinary expansions 
of $n$ by $\mathbb{H}_{n}$, the number of powers that are used exactly twice in the hyperbinary expansion $x \in \mathbb{H}_{n}$ by $\mathfrak{h}_{n}(x)$, and the total number of distinct powers that are used in the hyperbinary expansion $x \in \mathbb{H}_{n}$ by $\mathfrak{d}_{n}(x)$. The $(p, q)$-hyperbinary expansion of $x$ is defined as $p^{\mathfrak{d}_{n}(x)} q^{\mathfrak{h}_{n}(x)}$.

Definition $2.3((p, q)$-Calkin-Wilf sequence). Let $f(n ; p, q)$ be the polynomial consisting of the sum of $(p, q)$-hyperbinary expansions of $n$ with $f(0 ; p, q)=1$ and $f(-1 ; p, q)=0$. Then we will call the sequence

$$
\begin{aligned}
\{f(n ; p, q)\}_{n \in \mathbb{N}_{0}}= & 1, p, p+p q, p^{2}, p+\left(p+p^{2}\right) q, p^{2}(1+q), \\
& p^{2}\left(1+q+q^{2}\right), p^{3}, p+\left(p+p^{2}+p^{3}\right) q, \ldots
\end{aligned}
$$

the $(p, q)$-hyperbinary sequence and call the sequence

$$
\begin{aligned}
\left\{\frac{f(n ; p, q)}{f(n+1 ; p, q)}\right\}_{n \in \mathbb{N}_{0}}= & \frac{1}{p}, \frac{p}{p(1+q)}, \frac{p(1+q)}{p^{2}}, \frac{p^{2}}{p+\left(p+p^{2}\right) q}, \\
& \frac{p+\left(p+p^{2}\right) q}{p^{2}(1+q)}, \frac{p^{2}(1+q)}{p^{2}\left(1+q+q^{2}\right)}, \ldots
\end{aligned}
$$

the $(p, q)$-Calkin-Wilf sequence.

Example 2.1. The hyperbinary expansions of 6 are $4+2,4+1+1$ and $2+2+1+1$. Thus, the $(p, q)$-hyperbinary expansions of 6 are $p^{2} q^{0}, p^{2} q$ and $p^{2} q^{2}$ and, accordingly, $f(6 ; p, q)=p^{2}+p^{2} q+p^{2} q^{2}$.

Following $[3,4]$, we may define branches and diagonals of the $(p, q)$ Calkin-Wilf tree as below.

Definition 2.4 (Branches and diagonals). Let $v$ be any vertex of the $(p, q)$ Calkin-Wilf tree and $j \in \mathbb{N}$. The set of all vertices that is generated when an infinite number of right (left) movements proceeding from the left (right) child of $v$ will be denoted by $\mathbb{L}_{v}\left(\mathbb{R}_{v}\right)$ and will be called the left (right) branch of $v$. The set $\mathbb{L}_{v}\left(\mathbb{R}_{v}\right)$ includes the left (right) child of $v$.

The $j$-th left (right) diagonal $L_{j}\left(R_{j}\right)$ of the $(p, q)$-Calkin-Wilf tree is the set of all vertices found at the $j$-th leftmost (rightmost) position in each level of the tree beginning at level $\left\lceil\log _{2} j\right\rceil+1$.

Example 2.2. We have

$$
\begin{aligned}
L_{1} & =\left\{\frac{p^{j-1}}{p+\left(p+p^{2}+\cdots+p^{j-1}\right) q}\right\}_{j \geq 1}, \\
\mathbb{R}_{\frac{1}{p}} & =\left\{\frac{p^{j-1}(1+q)}{p^{2}+\left(p^{2}+p^{3}+\cdots+p^{j-1}\right)\left(q+q^{2}\right)}\right\}_{j \geq 2},
\end{aligned}
$$




$$
\begin{aligned}
& R_{1}=\left\{\frac{p^{j-1}\left(1+q+\cdots+q^{j-1}\right)}{p^{j}}\right\}_{j \geq 1}, \\
& L_{2}=\left\{\frac{p+\left(p+p^{2}+\cdots+p^{j-1}\right) q}{p^{2}+\left(p^{2}+p^{3}+\cdots p^{j-1}\right) q}\right\}_{j \geq 2},
\end{aligned}
$$

and

$$
R_{2}=\left\{\frac{p^{j-1}\left(1+q+\cdots+q^{j-2}\right)}{p^{j-1}\left(1+q+\cdots+q^{j-1}\right)}\right\}_{j \geq 2} .
$$

A proof of the following theorem when $p=q=1$ can be found in [5] and an inductive proof is given in Aigner and Ziegler [1]. Recently, Bates et al. [3] offered another proof based on branching in the tree which can be readily generalized to establish the following theorem for the $(p, q)$-Calkin-Wilf tree.

Theorem 2.1. Let the concatenation of successive levels of the $(p, q)$-CalkinWilf tree form a sequence. Then the denominator of the $i$-th term of this sequence is the numerator of the $(i+1)$-st term for all $i$.

Remark. The sequence described in Theorem 2.1 is of the form

$$
\left\{\frac{g(n ; p, q)}{g(n+1 ; p, q)}\right\}_{n \in \mathbb{N}_{0}}
$$

for some function $g$ with $g(0 ; p, q)=1$ and $g(1 ; p, q)=p$. The left and right children of

$$
\left\{\frac{g(n ; p, q)}{g(n+1 ; p, q)}\right\}
$$

are, respectively,

$$
\left\{\frac{g(2 n+1 ; p, q)}{g(2 n+2 ; p, q)}\right\} \quad \text { and } \quad\left\{\frac{g(2 n+2 ; p, q)}{g(2 n+3 ; p, q)}\right\} .
$$

The next result shows how the $(p, q)$-Calkin-Wilf sequence is related to the $(p, q)$-Calkin-Wilf tree.

Theorem 2.2. The $(p, q)$-Calkin-Wilf sequence is the concatenation of successive levels of the $(p, q)$-Calkin-Wilf tree. That is, for all $n \in \mathbb{N}_{0}$,

$$
g(n ; p, q)=f(n ; p, q)
$$


Proof. By the preceding remark and Definition 2.1, we obtain the recurrences

$$
\begin{aligned}
& g(2 n+1 ; p, q)=p g(n ; p, q) \\
& g(2 n+2 ; p, q)=p q g(n ; p, q)+g(n+1 ; p, q)
\end{aligned}
$$

for all $n \in \mathbb{N}_{0}$, with $g(0 ; p, q)=1$. Now suppose $x$ is any hyperbinary expansion of the integer $m$. If $m=2 n+1$, then the hyperbinary expansion $x$ must contain exactly one power of $2^{0}=1$. Subtracting this and reducing all of the other powers of 2 by one in $x$ gives a hyperbinary expansion of the integer $n$ (note that all such expansions of $n$ arise uniquely in this manner). If $m=2 n+2$, then $x$ either contains no copies of the power $2^{0}$ (in which case, simply reduce each power of 2 by one) or exactly two copies of $2^{0}$ (in which case, first subtract 2 before dividing by it). This yields the following set of recurrences:

$$
\begin{aligned}
& f(2 n+1 ; p, q)=p f(n ; p, q) \\
& f(2 n+2 ; p, q)=f(n+1 ; p, q)+p q f(n ; p, q)
\end{aligned}
$$

for all $n \in \mathbb{N}_{0}$, with $f(0 ; p, q)=1$. The result now follows from (2) and (3).

Definition 2.5. The generating function for the $(p, q)$-hyperbinary sequence $\{f(n ; p, q)\}_{n \geq 0}$ is defined by

$$
F(x, p, q)=\sum_{n \geq 0} f(n ; p, q) x^{n} .
$$

Theorem 2.3. The generating function $F(x, p, q)$ is given by

$$
F(x, p, q)=\prod_{j \in \mathbb{N}_{0}}\left(1+p x^{2^{j}}+p q x^{2^{j+1}}\right) .
$$

Proof. By recurrence (3), we have

$$
\begin{aligned}
F(x, p, q)= & 1+\sum_{n \geq 0} f(2 n+1 ; p, q) x^{2 n+1}+\sum_{n \geq 0} f(2 n+2 ; p, q) x^{2 n+2} \\
= & 1+p \sum_{n \geq 0} f(n ; p, q) x^{2 n+1}+\sum_{n \geq 0} f(n+1 ; p, q) x^{2 n+2} \\
& +p q \sum_{n \geq 0} f(n ; p, q) x^{2 n+2}
\end{aligned}
$$




$$
=\left(1+p x+p q x^{2}\right) \sum_{n \geq 0} f(n ; p, q) x^{2 n}
$$

which implies

$$
\begin{aligned}
F(x, p, q) & =\sum_{n \geq 0} f(n ; p, q) x^{n}=\left(1+p x+p q x^{2}\right) F\left(x^{2}, p, q\right) \\
& =\left(1+p x+p q x^{2}\right)\left(1+p x^{2}+p q x^{4}\right) F\left(x^{4}, p, q\right) \\
& =\cdots \\
& =\prod_{j \in \mathbb{N}_{0}}\left(1+p x^{2^{j}}+p q x^{2^{j+1}}\right),
\end{aligned}
$$

as required.

One may provide explicit formulas for the sequences of left and right descendants starting from any point.

Theorem 2.4. We have

$$
\mathbb{L}_{\frac{a}{b}}=\left\{\frac{p^{k-1}(p q a+b)\left(1+q+\cdots+q^{k-1}\right)+p^{k+1} q^{k} a}{p^{k}(p q a+b)}\right\}_{k \geq 0}
$$

and

$$
\mathbb{R}_{\frac{a}{b}}=\left\{\frac{p^{k}(p q a+b)}{p b+q(p q a+b)\left(p+p^{2}+\cdots+p^{k}\right)}\right\}_{k \geq 0} .
$$

Proof. From Definitions 2.1 and 2.4, we have

$$
\mathbb{L}_{\frac{a}{b}}=\frac{p a}{p q a+b}, \frac{p^{2} q a+p q a+b}{p^{2} q a+p b}, \frac{p^{3} q^{2} a+p^{2} q^{2} a+p q b+p^{2} q a+p b}{p^{3} q a+p^{2} b}, \ldots,
$$

and, by induction, the $k$-th term of this sequence, $k \geq 1$, is given by

$$
\frac{\left(p^{k-1} q a+p^{k-2} b\right)\left(1+q+\cdots+q^{k-2}\right)+p^{k} q^{k-1} a}{p^{k} q a+p^{k-1} b} .
$$

Replacing $k$ with $k+1$ gives the first result. Similarly,

$$
\mathbb{R}_{\frac{a}{b}}=\frac{p q a+b}{p b}, \frac{p^{2} q a+p b}{p^{2} q^{2} a+p q b+p b}, \frac{p^{3} q a+p^{2} b}{p^{3} q^{2} a+p^{2} q b+p^{2} q^{2} a+p q b+p b}, \ldots,
$$

whose $k$-th term, $k \geq 1$, is given by

$$
\frac{p^{k} q a+p^{k-1} b}{p b+\left(p q^{2} a+q b\right)\left(p+p^{2}+\cdots+p^{k-1}\right)} .
$$


We have the following partial result for succession.

Theorem 2.5. Let $x_{n}$ be the $n$-th term in the $(p, q)$-Calkin-Wilf sequence where $n \in \mathbb{N}$. Then

$$
x_{2 n+1}=\frac{1}{p-p q x_{2 n}} .
$$

Proof. By Definition 2.3 and the recurrence (3), we have

$$
x_{2 n}=\frac{f(2 n-1 ; p, q)}{f(2 n ; p, q)}=\frac{p f(n-1 ; p, q)}{p q f(n-1 ; p, q)+f(n ; p, q)},
$$

and so

$$
\begin{aligned}
x_{2 n+1} & =\frac{f(2 n ; p, q)}{f(2 n+1 ; p, q)} \\
& =\frac{p q f(n-1 ; p, q)+f(n ; p, q)}{p f(n ; p, q)} \\
& =\frac{p q f(n-1 ; p, q)+f(n ; p, q)}{p(p q f(n-1 ; p, q)+f(n ; p, q))-p^{2} q f(n-1 ; p, q)} \\
& =\frac{1}{p-p q \frac{p f(n-1 ; p, q)}{p q f(n-1 ; p, q)+f(n ; p, q)}} \\
& =\frac{1}{p-p q x_{2 n}}
\end{aligned}
$$

as claimed.

Before we state our next result concerning succession, we will need the following lemma.

Lemma 2.6. Suppose $q \geq p>0$ are fixed real numbers and that $x \in \mathbb{R}^{+}$ can be expressed in the form

$$
x=\frac{1}{p}\left(1+q+\cdots+q^{k-1}\right)+\epsilon q^{k-1},
$$

for some $k \in \mathbb{N}_{0}$ and $\epsilon \in[0,1)$. Then $k$ and $\epsilon$ are uniquely determined.

Proof. Suppose, to the contrary, that we may express $x$ in the form (4) for some $k_{1}, \epsilon_{1}$ and $k_{2}, \epsilon_{2}$, where $k_{1}>k_{2} \geq 0$. Equating expressions for $x$ implies

$$
\frac{1}{p}\left(q^{k_{2}}+\cdots+q^{k_{1}-1}\right)=\epsilon_{2} q^{k_{2}-1}-\epsilon_{1} q^{k_{1}-1} .
$$


On the other hand, we have

$$
\epsilon_{2} q^{k_{2}-1}-\epsilon_{1} q^{k_{1}-1} \leq \epsilon_{2} q^{k_{2}-1}=\frac{\epsilon_{2}}{q} q^{k_{2}}<\frac{1}{p} q^{k_{2}} \leq \frac{1}{p}\left(q^{k_{2}}+\cdots+q^{k_{1}-1}\right),
$$

which contradicts (5).

Given $x \in \mathbb{R}^{+}$of the form in (4), let $k_{x}=k_{x}^{(p, q)}$ denote the uniquely determined member of $\mathbb{N}_{0}$. We can now state a full recurrence for the $(p, q)$ Calkin-Wilf sequence when $q \geq p>0$.

Theorem 2.7. Let $x_{n}$ be the $n$-th term in the $(p, q)$-Calkin-Wilf sequence, where $n \geq 2$ and $q \geq p>0$ are fixed real numbers. If $x_{n}$ belongs to $L_{1}$, then

$$
x_{n}= \begin{cases}\frac{1}{1+x_{n-1}}, & \text { if } q=p=1 ; \\ \frac{p^{\left(p x_{n-1}-1\right)}(p-1)}{p+p^{p x} n-1-2}, & \text { if } q=1 \text { and } p \neq 1 \\ \frac{1}{1+t q}, & \text { if } q \neq 1 \text { and } p=1 \\ \frac{p^{t-1}(p-1)}{p-1+\left(p^{t}-1\right) q}, & \text { if } q \neq 1 \text { and } p \neq 1,\end{cases}
$$

where $t=\frac{\log \left(1+p(q-1) x_{n-1}\right)}{\log (q)}$ in the last two cases. If $x_{n}$ does not belong to $L_{1}$ and $k=k_{x_{n-1}}$ is as defined above, then

$$
x_{n}=\left\{\frac{1}{p^{k-1}}+q \sum_{i=0}^{k-1} \frac{1}{p^{i}}+\frac{1}{p^{k}} \sum_{i=0}^{k-1} \frac{1}{q^{i}}-\frac{x_{n-1}}{(p q)^{k-1}}\right\}^{-1} .
$$

Proof. Suppose $x_{n} \in L_{1}$ and that it is the leftmost member of level $j+1$ for some $j \geq 1$, which implies $x_{n-1}$ is the rightmost member of level $j$. Thus, we have

$$
x_{n-1}=\frac{1+q+\cdots+q^{j-1}}{p}
$$

and

$$
x_{n}=\frac{p^{j-1}}{1+\left(1+p+\cdots+p^{j-1}\right) q} .
$$

If $q=1$, then $x_{n-1}=\frac{j}{p}$ and the first two cases follow upon considering whether or not $p$ is one. If $q \neq 1$, then

$$
x_{n-1}=\frac{q^{j}-1}{p(q-1)},
$$


which implies $j=\frac{\log \left(1+p(q-1) x_{n-1}\right)}{\log (q)}$. Now substitute $j$ into the expression for $x_{n}$, differentiating the cases when $p=1$ and $p \neq 1$.

Now suppose $x_{n}$ does not belong to $L_{1}$. Let $\frac{a}{b}$ denote the closest common ancestor of $x_{n-1}$ and $x_{n}$. Then $x_{n-1}$ and $x_{n}$ are the $\ell$-th terms in the left and right branches, respectively, of $\frac{a}{b}$ for some $\ell \in \mathbb{N}_{0}$. From Theorem 2.4, we have

$$
x_{n-1}=\frac{p^{\ell-1}(p q a+b)\left(1+q+\cdots+q^{\ell-1}\right)+p^{\ell+1} q^{\ell} a}{p^{\ell}(p q a+b)}
$$

and

$$
x_{n}=\frac{p^{\ell}(p q a+b)}{p b+p q(p q a+b)\left(1+p+\cdots+p^{\ell-1}\right)} .
$$

Rewrite $x_{n-1}$ as

$$
x_{n-1}=\frac{1}{p}\left(1+q+\cdots+q^{\ell-1}\right)+\left(\frac{p q a}{p q a+b}\right) q^{\ell-1} .
$$

From (7) and Lemma 2.6 (take $\epsilon=\frac{p q a}{p q a+b}$ ), we see that $\ell=k_{x_{n-1}}$, which we'll denote simply by $k$. Therefore, we have

$$
\begin{aligned}
\frac{1}{x_{n}}= & q\left(1+\frac{1}{p}+\cdots+\frac{1}{p^{k-1}}\right)+\frac{1}{p^{k-1}}\left(\frac{b}{p q a+b}\right) \\
= & q\left(1+\frac{1}{p}+\cdots+\frac{1}{p^{k-1}}\right)+\frac{1}{p^{k-1}}\left(1-\frac{p q a}{p q a+b}\right) \\
= & q\left(1+\frac{1}{p}+\cdots+\frac{1}{p^{k-1}}\right) \\
& +\frac{1}{p^{k-1}}\left(1-\frac{x_{n-1}}{q^{k-1}}+\frac{1}{p}\left(1+\frac{1}{q}+\cdots+\frac{1}{q^{k-1}}\right)\right),
\end{aligned}
$$

by (7), which gives the result.

The proof above specializes to yield recurrence (1) satisfied by the CalkinWilf sequence.

Corollary 2.8. If $x_{n}$ denotes the $n$-th term of the Calkin-Wilf sequence, then

$$
x_{n}=\frac{1}{2\left\lfloor x_{n-1}\right\rfloor+1-x_{n-1}}, \quad n \geq 2,
$$

with initial condition $x_{1}=1$. 
Proof. First suppose $x_{n}$ does not belong to $L_{1}$. If $p=q=1$, we have $k=k_{x_{n-1}}=\left\lfloor x_{n-1}\right\rfloor$, by definition. Theorem 2.7 when $p=q=1$ then implies

$$
x_{n}=\frac{1}{2 k+1-x_{n-1}}=\frac{1}{2\left\lfloor x_{n-1}\right\rfloor+1-x_{n-1}} .
$$

If $x_{n} \in L_{1}$, then $x_{n-1}=j$ and $x_{n}=\frac{1}{j+1}$ for some $j \geq 1$ and the recurrence holds in this case as well.

We have the following partial result concerning the uniqueness of the entries of the $(p, q)$-Calkin-Wilf tree. We refer to the $p=1$ case as the $q$-Calkin-Wilf tree.

Theorem 2.9. If $q$ is a positive integer, then the entries of the q-CalkinWilf tree are distinct positive rational numbers in lowest terms.

Proof. First note that if $\frac{a}{b}$ is a fraction in lowest terms, then so are its children, $\frac{a}{q a+b}$ and $\frac{q a+b}{b}$, which implies all of the entries are positive rational numbers in lowest terms by induction. We next treat the uniqueness. First note that the members of $R_{1}$ (which are of the form $1+q+\cdots+q^{i}$ ) are distinct from all of the other entries of the tree, since they are integers with no other entries of the tree integral (recall all fractions are in lowest terms). To show that all of the entries in the tree are distinct, we will prove that all of the entries in the first $n$ rows are distinct for each $n \geq 1$, by induction on $n$, the $n=1$ and $n=2$ cases clear (note that the numbers $1, \frac{1}{q+1}$ and $q+1$ are distinct).

Let us assume the statement holds for $n$ and prove it for $n+1$. Suppose, to the contrary, that it fails for $n+1$, where $n \geq 2$, and that $x=y$, where $x$ is an entry in row $n+1$ and $y$ is another entry in row $k$ for some $k \leq n+1$. We may also assume further that neither $x$ nor $y$ belongs to $R_{1}$. Given $z \notin R_{1}$, let $\bar{z}$ be an entry such that $z \in \mathbb{L}_{\bar{z}}$; we show that $\bar{z}$ is uniquely determined. If $\bar{z}=\frac{a}{b}$, written in lowest terms, then the $p=1$ case of Theorem 2.4 implies $z$ is expressible as

$$
z=\left(1+q+\cdots+q^{r-2}\right)+q^{r-2}\left(\frac{q a}{q a+b}\right)
$$

for some $r \geq 1$. By Lemma 2.6, note that $r$, and hence $a, b$, and $\bar{z}$, are uniquely determined by the numerical value of $z$. (Indeed, one can show further that the mapping $z \mapsto \bar{z}$ is a bijection from the $2^{n}-1$ entries comprising row $n+1$ excepting the rightmost entry to the $2^{n}-1$ entries comprising the first $n$ rows for each $n \geq 1$, by an inductive argument.) 
Thus, the number of rows $r$ one must go back along the tree to reach $\bar{z}$ starting from $z$ is determined by $z$. So $x=y$ implies $\bar{x}=\bar{y}$, with $\bar{x}$ and $\bar{y}$ corresponding to distinct positions in rows $n+1-r$ and $k-r$, respectively, for some $r \geq 1$, which contradicts the induction hypothesis. This completes the induction step and implies that all of the entries of the tree are distinct.

Remark. Not all of the positive rationals appear in the $q$-Calkin-Wilf tree for integers $q>1$, which is apparent since not all positive integers are of the form $1+q+\cdots+q^{i}$ in this case.

\section{The $q$-Calkin-Wilf $k$-tree and sequence}

The following definition of the $q$-Calkin-Wilf $k$-tree provides another generalization of the one presented in Bates et al. [3] for the Calkin-Wilf tree.

Definition 3.1 ( $q$-Calkin-Wilf $k$-tree). The $q$-Calkin-Wilf $k$-tree is a $k$ ary tree whereby each vertex $\left(a_{1}, a_{2}, \ldots, a_{k}\right)$ has fixed dimension, $k$, and $(1,1, \ldots, 1)$ represents the root of the tree. Each vertex $\left(a_{1}, \ldots, a_{k}\right)$ is a parent to exactly $k$ children:

$$
\left(a_{1}, \ldots, a_{1}, q a_{1}+a_{2}\right),\left(a_{1}, \ldots, a_{1}, q a_{1}+a_{2}, a_{2}\right), \ldots,\left(q a_{1}+a_{2}, a_{2}, \ldots, a_{2}\right),
$$

going from the leftmost to the rightmost child, where $q$ may either be regarded as an indeterminate or as an element of $\mathbb{R}^{+}$. Each of these children is located one level below its parent on the tree.

Figure 3 shows the first three levels of the $q$-Calkin-Wilf 3 -tree.

By induction on $j$, the leftmost and rightmost vertices in level $j$ of the $q$-Calkin-Wilf $k$-tree are given, respectively, by

$$
(1, \ldots, 1,1+q) \quad \text { and } \quad\left(1+q+\cdots+q^{j-1}, 1, \ldots, 1\right) .
$$

Similarly, the second leftmost and second rightmost vertices in level $j$, where $j \geq 2$, are given, respectively, by

$$
(1, \ldots, 1,1+q, 1) \quad \text { and } \quad\left(1+q+\cdots+q^{j-2}, 1+2+\cdots+q^{j-1}, 1, \ldots, 1\right) .
$$

The left diagonals of the $q$-Calkin-Wilf $k$-tree assume a particularly simple form.

Proposition 3.1. If $k \geq 3$, then each left diagonal of the $q$-Calkin-Wilf $k$-tree, except for the first, has every entry identical. The first left diagonal has identical entries except for the first term. 


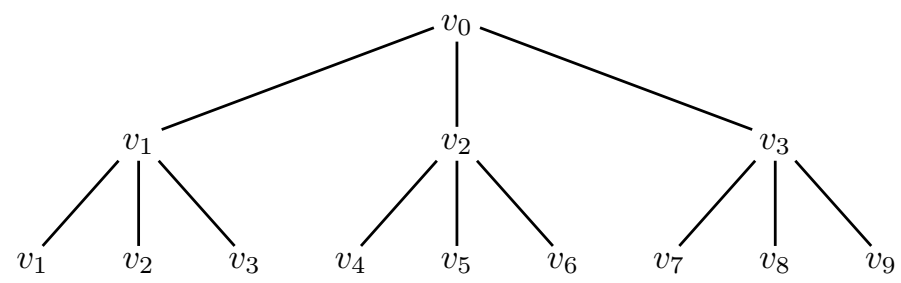

Figure 3: The first three levels of the $q$-Calkin-Wilf 3 -tree, where $v_{0}=$ $(1,1,1), v_{1}=(1,1, q+1), v_{2}=(1, q+1,1), v_{3}=(q+1,1,1), v_{4}=(1,1,2 q+1)$, $v_{5}=(1,2 q+1, q+1), v_{6}=(2 q+1, q+1, q+1), v_{7}=\left(q+1, q+1, q^{2}+q+1\right)$, $v_{8}=\left(q+1, q^{2}+q+1,1\right)$ and $v_{9}=\left(q^{2}+q+1,1,1\right)$.

Proof. Note that there are $k^{n-1}$ entries of level $n$ in the $q$-Calkin-Wilf $k$-tree for each $n \geq 1$. By induction, our result is an immediate consequence of the fact that the leftmost $k^{n-2}$ entries of level $n$ are identical to the entries of level $n-1$ for all $n \geq 2$. To see this, note that the first $k^{n-2}$ entries in level $n$ are all descendants of the first entry in the second row and therefore they correspond to the $(n-1)$-st row of the $q$-Calkin-Wilf $k$-tree with root $(1, \ldots, 1,1+q)$ instead of $(1, \ldots, 1)$ (which would have all the same entries except for the root).

Furthermore, note that writing the set of vectors representing the children of a node left-to-right in column form yields a symmetric matrix. In this matrix, the diagonal that proceeds from the bottom left to the upper right consists of identical entries.

The next two definitions provide a $q$-generalization for the number of hyper $k$-expansions of an integer $n$.

Definition 3.2 ( $q$-hyper $k$-expansions). A hyper $k$-expansion of a number $n$ is an expansion of $n$ as a sum of powers of $k$, each power being used at most $k$ times. We denote the set of all hyper $k$-expansions of $n$ by $\mathbb{H}_{k}(n)$ and the total number of powers that are used exactly $k$ times in the hyper $k$-expansion $x \in \mathbb{H}_{k}(n)$ by $\mathfrak{h}_{k, n}(x)$. The $q$-hyper $k$-expansion of $x$ is defined as $q^{\mathfrak{h}_{k, n}(x)}$.

Definition 3.3 ( $q$-Calkin-Wilf $k$-sequence). Define $f_{k}(n ; q)$ to be the polynomial for the sum of all $q$-hyper $k$-expansions of $n \in \mathbb{N}$, with $f_{k}(0 ; q)=1$. The $f_{k}(n ; q)$ will be called $q$-hyper $k$-expansion polynomials. We will call the sequence of vectors

$$
\left\{v_{k}(n)\right\}_{n \in \mathbb{N}_{0}}=\left\{\left(f_{k}(n ; q), f_{k}(n+1 ; q), \ldots, f_{k}(n+k-1 ; q)\right)\right\}_{n \in \mathbb{N}_{0}}
$$

the $q$-Calkin-Wilf $k$-sequence. 
Note that the sequence $\left\{v_{2}(n)\right\}_{n \in \mathbb{N}_{0}}$ provides an alternate representation of the $(p, q)$-Calkin-Wilf sequence presented in Definition 2.3 above in the case when $p=1$.

Example 3.1. The hyper 3-expansions of 12 are $3^{2}+3,3^{2}+1+1+1$ and $3+3+3+1+1+1$. Thus, the $q$-hyper 3 -expansions of 12 are $q^{0}, q^{1}$ and $q^{2}$ and, accordingly, $f_{3}(12 ; q)=q^{2}+q+1$. Similarly, the $q$-hyper 3 -expansions of $n$ for $n=0,1,2, \ldots, 21$ are given by

$$
\begin{aligned}
& 1,1,1, q+1,1,1, q+1,1,1,2 q+1, q+1, q+1, q^{2}+q+1,1,1, q+1,1,1 \\
& \quad 2 q+1, q+1, q+1
\end{aligned}
$$

The next result shows how the $q$-Calkin-Wilf $k$-tree is related to the $q$-Calkin-Wilf $k$-sequence.

Theorem 3.2. If $k \geq 2$, then let the concatenation of successive levels of the $q$-Calkin-Wilf $k$-tree form a sequence $u_{k}(n ; q)$ of vectors of length $k$. Then

$$
u_{k}(n)=v_{k}(n)
$$

for all $n \geq 0$.

Proof. From Definition 3.1, the children of the vertex $u_{k}(n)$ in the $q$-CalkinWilf $k$-tree are given by

$$
u_{k}(k n+1), u_{k}(k n+2), \ldots, u_{k}(k n+k)
$$

Write $u_{k}(n)=\left(g_{k}(n ; q), g_{k}(n+1 ; q), \ldots, g_{k}(n+k-1 ; q)\right)$ for all $n \in \mathbb{N}_{0}$. From Definition 3.1, we have

$$
\begin{aligned}
& g_{k}(k n+j ; q)=g_{k}(n ; q), \quad \text { for all } j=1,2, \ldots, k-1, \\
& g_{k}(k n+k ; q)=g_{k}(n+1 ; q)+q g_{k}(n ; q)
\end{aligned}
$$

for all $n \in \mathbb{N}_{0}$, with $g_{k}(0 ; q)=1$. Now suppose $x$ is any hyper $k$-expansion of the integer $m$. If $m=k n+j$ for some $j, 1 \leq j \leq k-1$, then the expansion $x$ must contain exactly $j$ powers of $k^{0}=1$. Subtracting $j$ and reducing all of the other powers of $k$ by one in $x$ gives a hyper $k$-expansion of the integer $n$. If $m=k n+k$, then $x$ either contains no powers of $k^{0}$ (in which case, simply reduce each power of $k$ by one) or exactly $k$ powers of $k^{0}$ (in which case, first subtract $k$ before dividing by it). This yields the following set of recurrences: 


$$
\begin{aligned}
& f_{k}(k n+j ; q)=f_{k}(n ; q), \quad \text { for all } j=1,2, \ldots, k-1, \\
& f_{k}(k n+k ; q)=f_{k}(n+1 ; q)+q f_{k}(n ; q)
\end{aligned}
$$

for all $n \in \mathbb{N}_{0}$, with $f_{k}(0 ; q)=1$. The result follows from (9) and (10).

In particular, we see that the sequence consisting of the first components of the vectors which label the vertices in the $q$-Calkin-Wilf $k$-tree is precisely the sequence $f_{k}(n ; q)$. These polynomials may be given explicitly in several cases.

Theorem 3.3. If $k \geq 2$, then

(i) $f_{k}\left(k^{m}-1 ; q\right)=1, \quad m \geq 1$,

(ii) $f_{k}\left(k^{m} ; q\right)=1+m q, \quad m \geq 1$,

(iii) $f_{k}\left(k^{m}+k ; q\right)=1+(m-1) q+(m-1) q^{2}, \quad m \geq 2$.

Proof. (i) Since $k^{m}-1=(k-1)\left(k^{m-1}+\cdots+k\right)+k-1$, then from (10), we have

$$
f_{k}\left(k^{m}-1 ; q\right)=f_{k}\left((k-1)\left(k^{m-2}+\cdots+1\right) ; q\right)=f_{k}\left(k^{m-1}-1 ; q\right),
$$

for all $m \geq 1$, whence the result follows from the fact $f_{k}(k-1 ; q)=1$.

(ii) Applying (10) when $n=k^{m}$, we have $f_{k}(n ; q)=f_{k}\left(k^{m-1} ; q\right)+$ $q f_{k}\left(k^{m-1}-1 ; q\right)$. Thus, by (i), we have $f_{k}\left(k^{m} ; q\right)=f_{k}\left(k^{m-1} ; q\right)+q$, with the initial condition $f_{k}(1 ; q)=1$, whence the result follows by induction on $m$.

(iii) If $m \geq 2$, then from (10),

$$
\begin{aligned}
f_{k}\left(k^{m}+k ; q\right) & =f_{k}\left(k^{m-1}+1 ; q\right)+q f_{k}\left(k^{m-1} ; q\right) \\
& =f_{k}\left(k^{m-2} ; q\right)+q(1+(m-1) q) \\
& =1+(m-2) q+q(1+(m-1) q),
\end{aligned}
$$

by (ii).

We next find an explicit formula for the ordinary generating function of the sequence $f_{k}(n ; q)$, where $k \geq 2$ is fixed.

Theorem 3.4. The generating function $F_{k}(x, q)=\sum_{n \geq 0} f_{k}(n ; q) x^{n}$ is given by

$$
F(x, q)=\prod_{j \in \mathbb{N}_{0}}\left(\frac{1-x^{k^{j+1}}}{1-x^{k^{j}}}+q x^{k^{j+1}}\right) .
$$


Proof. By (10), we have

$$
\begin{aligned}
F_{k}(x, q) & =1+\sum_{n \geq 0} \sum_{j=1}^{k} f_{k}(k n+j ; q) x^{k n+j} \\
& =1+\sum_{n \geq 0} \sum_{j=1}^{k-1} f_{k}(n ; q) x^{k n+j}+\sum_{n \geq 0}\left[f_{k}(n+1 ; q)+q f_{k}(n ; q)\right] x^{k n+k} \\
& =\sum_{j=0}^{k-1} x^{j} F_{k}\left(x^{k}, q\right)+q x^{k} F_{k}\left(x^{k}, q\right) .
\end{aligned}
$$

Therefore, we have

$$
\begin{aligned}
F_{k}(x, q) & =\left(\frac{1-x^{k}}{1-x}+q x^{k}\right) F_{k}\left(x^{k}, q\right) \\
& =\left(\frac{1-x^{k}}{1-x}+q x^{k}\right)\left(\frac{1-x^{k^{2}}}{1-x^{k}}+q x^{k^{2}}\right) F_{k}\left(x^{k^{2}}, q\right) \\
& =\cdots \\
& =\prod_{j \in \mathbb{N}_{0}}\left(\frac{1-x^{k^{j+1}}}{1-x^{k^{j}}}+q x^{k^{j+1}}\right),
\end{aligned}
$$

as required.

When $q=0$, the product in Theorem 3.4 telescopes to give

$$
F_{k}(x, 0)=\frac{1}{1-x}
$$

in accordance with the uniqueness of the usual $k$-ary expansion. We conclude by describing a recurrence satisfied by the sequence of fractions $x_{k}(n):=$ $\frac{f_{k}(n+1 ; q)}{f_{k}(n+2 ; q)}$.

Theorem 3.5. If $n \geq 1$, then

$$
\begin{aligned}
x_{k}(k n+k-1) & =1+q x_{k}(n-1), \\
x_{k}(k n+k-2) & =\frac{x_{k}(n-1)}{1+q x_{k}(n-1)}, \\
x_{k}(k n+j) & =1,
\end{aligned}
$$

for all $j=0,1,2, \ldots, k-3$. 
Proof. By (10), we have

$$
\begin{aligned}
x_{k}(k n+d-1) x_{k}(k n+d) \cdots x_{k}(k n+k-2) & =\frac{f_{k}(k n+d ; q)}{f_{k}(k n+k ; q)} \\
& =\frac{f_{k}(n ; q)}{f_{k}(n+1 ; q)+q f_{k}(n)} \\
& =\frac{x_{k}(n-1)}{1+q x_{k}(n-1)},
\end{aligned}
$$

for all $d \in\{1,2, \ldots, k-1\}$. Applying this equation for $d=k-1, k-2, \ldots, 1$, we obtain

$$
x_{k}(k n+k-2)=\frac{x_{k}(n-1)}{1+q x_{k}(n-1)}
$$

and

$$
x_{k}(k n)=\cdots=x_{k}(k n+k-3)=1 .
$$

Also, by (10), we get

$$
\begin{aligned}
x_{k}(k n+k-1) & =\frac{f_{k}(k n+k ; q)}{f_{k}(k n+k+1 ; q)} \\
& =\frac{f_{k}(n+1 ; q)+q f_{k}(n ; q)}{f_{k}(n+1 ; q)} \\
& =1+q x_{k}(n-1 ; q),
\end{aligned}
$$

as claimed.

\section{Acknowledgment}

We wish to thank Bruce Bates for useful discussions and for several suggestions which improved the presentation of this paper.

\section{References}

[1] M. Aigner and G. M. Ziegler, Proofs from the Book, Springer, 2009.

[2] G. Alkauskas and J. Steuding, Statistical properties of the Calkin-Wilf tree: Real and $p$-adic distribution, arXiv:0801.0054.

[3] B. Bates, M. Bunder, and K. Tognetti, Linking the Calkin-Wilf and Stern-Brocot trees, European J. Combin. 31 (2010) 1637-1661. MR2673006 
[4] B. Bates and T. Mansour, The $q$-Calkin-Wilf tree, J. Combin. Theory Ser. A 118 (2011) 1143-1151. MR2763063

[5] N. Calkin and H. S. Wilf, Recounting the rationals, Amer. Math. Monthly 107 (2000) 360-363. MR1763062

[6] A. J. Cole and A. J. Davie, A game based on the Euclidean algorithm and a winning strategy for it, Math. Gaz. 53 (1969) 354-357. MR0258472

[7] S. Hofmann, G. Schuster, and J. Steuding, Euclid, Calkin \& Wilf playing with rationals, Elem. Math. 63 (2008) 109-117. MR2424893

[8] D. E. Knuth, Problem 10906, American Mathematical Monthly; Solution by Moshe Newman, Amer. Math. Monthly 110 (2003) 642-643.

[9] B. Reznick, Regularity properties of the Stern enumeration of the rationals, J. Integer Seq. 11 (2008) Article 08.4.1, 17pp. MR2447843

[10] M. A. Stern, Über eine zahlentheoretische funktion, J. Reine Angew. Math. 55 (1858) 193-220.

TOUfIK Mansour

Department of Mathematics

UNIVERSITY OF HAIFA

31905 HAIFA

ISRAEL

E-mail address: tmansour@univ.haifa.ac.il

Mark Shattuck

DEPARTMENT OF MATHEMATiCS

UNIVERSity OF TENNESSEE

KNOXVILLE, TN 37996

USA

E-mail address: shattuck@math.utk. edu

Received December 6, 2010 